\title{
Current Management Strategies for the Prevention and Treatment of Cytomegalovirus Infection in Pediatric Transplant Recipients
}

\author{
Javier Bueno, ${ }^{1}$ Carmen Ramil ${ }^{1}$ and Michael Green ${ }^{2}$
}

1 Pediatric Transplantation Unit, Juan Canalejo Hospital, A Coruña, Spain

2 Division of Allergy, Immunology and Infectious Disease, Children's Hospital of Pittsburgh, Pittsburgh, Pennyslvania, USA

\section{Contents}

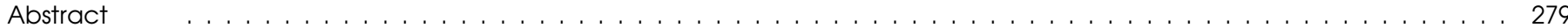

1. Epidemiology of Cytomegalovirus (CMV) Infection in Transplant Recipients . . . . . . . . . . . . . . . . . . . . . 280

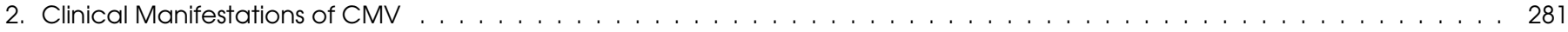

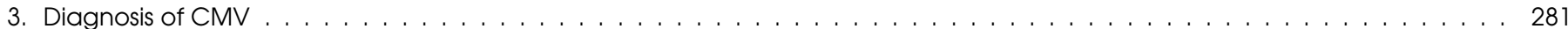

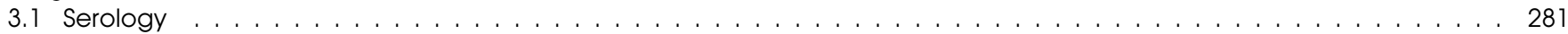

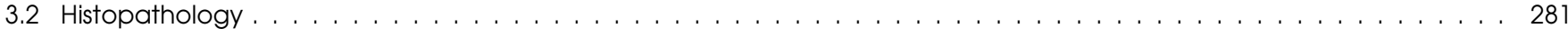

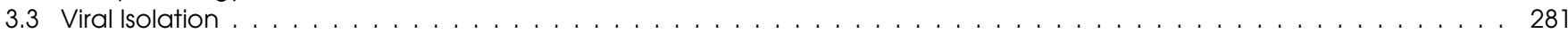

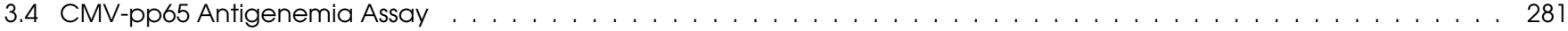

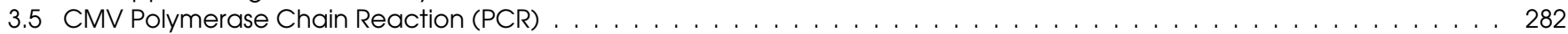



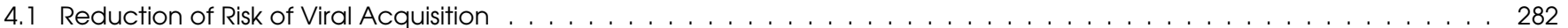

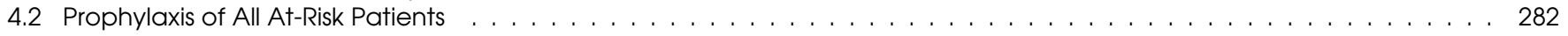

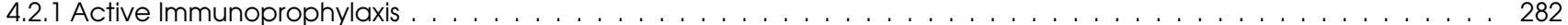

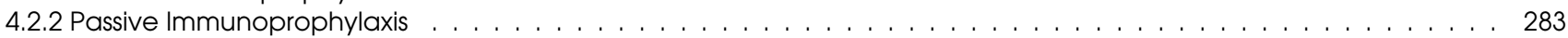

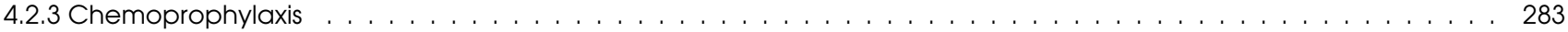

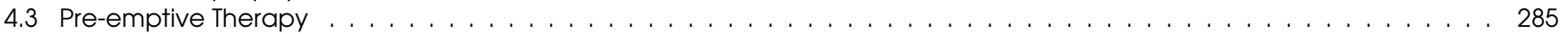

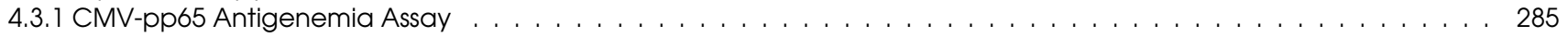

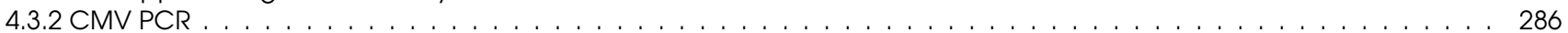

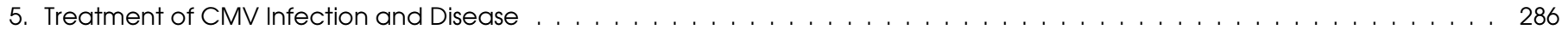

6. Conclusions $\ldots \ldots$

Abstract
Cytomegalovirus (CMV) is a significant cause of morbidity and mortality following transplantation, especially in the pediatric population, who remain at high risk of primary infection. The availability of effective antiviral therapy has led to dramatic improvements in the outcome of CMV infection in patients undergoing transplantation. In recent years, three major strategies have been developed for the prevention of CMV disease in this population: (i) reduction of risk of viral acquisition or reactivation by management of risk factors; (ii) prophylaxis of all 'at-risk' patients using prophylactic strategies for a defined period of time, initiated at or near the time of transplant; and (iii) pre-emptive treatment with ganciclovir of selected 'at-risk' patients, guided by either laboratory markers indicative of subclinical infection or the presence of specific risk factors.

In general, well designed comparative studies of one or more antiviral agents for the prevention of CMV have not been carried out. While ganciclovir appears to be more effective than aciclovir, its tolerability profile is less optimal. The use of foscarnet avoids myelosuppresions, but is associated with significant nephrotoxicity. Its use should be reserved for patients unable to tolerate ganciclovir or with ganciclovir-resistant CMV disease. Similar to foscarnet, the high frequency of nephrotoxicity associated with the use of cidofovir limits its use to clinical scenarios suggestive of ganciclovir resistance. Newer options, such as valaciclovir and valganciclovir, are currently under investigation and preliminary experience has been promising. Finally, passive immunoprophylaxis has been shown to prevent CMV disease after solid organ transplantation, but its use in bone marrow transplantation is controversial. 
Essentially, pre-emptive strategies have relied on the quantitation in the peripheral blood of CMV phosphoprotein pp65 antigen and/or the polymerase chain reaction assay. Strict guidelines for the use of those assays as a guide to pre-emptive therapy have not been standardized. Prospective trials comparing pre-emptive therapy using either intravenous or oral ganciclovir, and now oral valganciclovir or valaciclovir, are necessary to determine the relative cost effectiveness and efficacy of these alternative strategies. Finally, it remains controversial as to whether prophylaxis or pre-emptive therapy is the optimal strategy for preventing CMV disease. While a growing body of literature describes these approaches in adult transplant recipients, published experience in children has been much more limited.

Infection with cytomegalovirus (CMV) has long been recognized as a major cause of morbidity and mortality after solid organ transplantation (SOT) and bone marrow transplantation (BMT). ${ }^{[1-8]}$ Pediatric recipients are a high risk group for this complication as a considerable proportion of them are CMVseronegative and receive organs from CMV-seropositive donors. In the absence of preventive strategies, between 25 and $80 \%$ of SOT and allogeneic BMT recipients will develop CMV infection, with symptomatic disease developing in 8 to $41 \%$ of these patients. ${ }^{[2-8]}$ Further, recurrent episodes of CMV disease have been reported to occur in 6 to $59 \%$ of SOT recipients. ${ }^{[9]}$ Most of these studies have combined both children and adults. The incidence of CMV is much lower after autologous BMT and peripheral-blood stem cell transplantation than after allogeneic BMT. ${ }^{[10]}$

Although CMV infection continues to be an important cause of morbidity in transplant recipients, the use of ganciclovir for the treatment and prevention of CMV disease has led to a dramatic decline in CMV-associated mortality in these patients. This review discusses the current status of the treatment and prevention of CMV disease in children undergoing transplantation.

\section{Epidemiology of Cytomegalovirus (CMV) Infection in Transplant Recipients}

Children undergoing transplantation are at risk of developing either primary (first-time infection in a CMV-seronegative patient) or secondary (reactivation of latent virus or reinfection with a new strain) CMV infection. The major source of CMV in children experiencing primary infection after transplant is either the donor organ (including the possibility of transmission via passenger leukocytes that accompany the graft) or blood products. CMV infection is defined by the presence of viral replication (CMVpositive culture from any site or seroconversion in a previously seronegative patient) in the absence of any symptoms. CMV disease is defined by the presence of symptoms attributable to active CMV replication in a patient with a positive culture or histological evidence of CMV on a tissue biopsy in the absence of another pathogen to explain these symptoms.

Table I shows the principal risk factors associated with CMV infection and disease. Patients with primary CMV infection are at the greatest risk of developing CMV disease and experiencing $\mathrm{CMV}$-associated morbidity; thus, CMV-seronegative recipients of organs from CMV-seropositive donors (D+/R-) are at the highest risk of developing symptomatic infection with CMV. Secondary CMV infection is more frequently associated with asymptomatic infection. When secondary infection is symptomatic, the risk of developing invasive disease is significantly lower than for patients experiencing primary CMV infection. The type and intensity of immunosuppression also has a strong influence on the risk of developing CMV disease: the higher the levels of immunosuppression, the higher the risk. Different forms of immunosuppression used in organ transplantation affect different aspects of CMV infection; antilymphocyte antibodies [e.g. antithymocyte globulin, muromonab CD3 (OKT3)] and cytotoxic drugs enhance viral activation from latency whereas cyclosporin, tacrolimus and corticosteroids promote the persistence and spread of the virus by suppressing the host's antiviral immune responses. ${ }^{[11]}$ Also, a higher incidence of CMV disease has been described in recipients of kidney allografts receiving mycophenolate mofetil compared with those who received standard immunosuppressive regimens. ${ }^{[12]}$ Mycophenolate mofetil selectively suppresses the proliferation of $\mathrm{B}$ and $\mathrm{T}$ lymphocytes, and it may influence viral replication by altering the cytokine profile or modifying the down-regulation of adhesion molecules induced by CMV. ${ }^{[13]}$ Other factors, including rejection, systemic infection and inflammation have also been associated with an increased rate of CMV infection, presumably by reactivation of CMV from latency. ${ }^{[14]}$ Finally, the type of organ transplant has been associated with a variable risk of devel-

Table I. Risk factors for CMV acquisition after transplantation

CMV-seronegative recipients who receive organs from CMV-seropositive donors

Use of CMV-seropositive blood transfusion

Use of intensive immunosuppression with antilymphocyte antibodies

Viral load of transplanted organ: more pronounced in lungs, intestine and pancreas

Situation with cytokine release: infections, surgery, stress situations

Acute graft-versus-host disease (after BMT)

Total body irradiation before transplantation (after BMT)

BMT = bone marrow transplantation; $\mathbf{C M V}=$ cytomegalovirus. 
oping CMV disease. Recipients of intestinal and lung transplantation appear to be at a higher risk of developing CMV disease than patients undergoing other types of SOT. This enhanced risk is probably related to the higher viral load of the organ, and the higher levels of immunosuppression required to maintain these grafts compared with other types of SOT.

\section{Clinical Manifestations of CMV}

The peak incidence of CMV disease traditionally occurs 45 to 60 days after SOT and BMT. ${ }^{[2,5-8,15]}$ The range of clinically apparent effects of CMV in transplant recipients includes fever, leukopenia, thrombocytopenia and mild atypical lymphocytosis; invasive disease may also involve the liver, lungs, and gastrointestinal tract. CMV retinitis is uncommon after transplantation. Of interest, the allograft tends to be the most common site of tissue invasive disease. After BMT, pneumonia and gastrointestinal disease are the most common manifestations. In addition to these direct clinical effects, CMV has also been associated with a number of indirect effects. Active CMV infection is thought to increase the net state of immunosuppression, predisposing patients to opportunistic super-infections with bacteria, protozoa and fungi. ${ }^{[13,16]}$ It has also been suggested that CMV contributes to acute and chronic allograft rejection and injury, and to decreased long-term survival of SOT recipients. ${ }^{[14,17-22]}$ A history of CMV infection has been associated with an increased incidence of obliterative bronchiolitis after lung transplantation, ${ }^{[20]}$ progressive atherosclerosis after heart transplantation, ${ }^{[21]}$ and vanishing bile duct syndrome after liver transplantation. ${ }^{[22]}$ All of those entities are manifestations of chronic rejection. Whether CMV increases the risk for acute and chronic graft versus host disease after BMT remains controversial. ${ }^{[23,24]}$

\section{Diagnosis of CMV}

Diagnostic studies for CMV include serology, histology and virological tests, as well as antigenemia assays and polymerase chain reaction (PCR) techniques.

\subsection{Serology}

Serology is very important in defining the clinical risk from $\mathrm{CMV}$ at the time of transplantation; however, serology is a poor diagnostic tool after transplantation because of the immune response alteration.

\subsection{Histopathology}

Historically, CMV has been diagnosed by histology, with the visualization of cytomegalic inclusion bodies on tissue specimen.
In addition, the use of immunohistochemistry has increased the sensitivity of histological diagnosis. ${ }^{[25]}$ An in situ DNA hybridization technique is less sensitive but highly specific; ${ }^{[26]}$ however, histological diagnosis is limited by the need to use invasive procedures to obtain samples.

\subsection{Viral Isolation}

Conventional detection of CMV in clinical specimens has been by direct viral culture in human fibroblasts, with follow-up visual examination for cytopathic effects over a period of 14 to 28 days. This lengthy period of time required for diagnostic confirmation limits its clinical usefulness. With the development of the shell vial assay using a monoclonal antibody directed at the immediate-early viral antigen, CMV can be detected earlier (1 to 2 days) with high accuracy.

\subsection{CMV-pp65 Antigenemia Assay}

The CMV-specific lower-matrix phosphoprotein pp65 is produced and secreted at the site of active infection, and subsequently phagocytosed by leukocytes; thus, CMV-pp65 antigen is identifiable in the nuclei of peripheral polymorphonuclear cells of patients with active CMV infection but is not detectable in patients where CMV is in a latent state. ${ }^{[27]}$ The CMV-pp65 antigen is visualized and quantitated under direct microscopy by either immunoperoxidase staining or an indirect immunofluorescence assay. ${ }^{[27-29]}$ In contrast to viral cultures, the antigenemia assay has a short processing time ( 3 to 5 hours) and a high diagnostic accuracy (being more sensitive than shell vial culture), and stays positive longer than culture after the institution of antiviral therapy. ${ }^{[15,30,31]}$ Elevated levels of the antigen are associated with the presence of, or progression to, CMV disease; the higher the level the greater the risk of disease or viremia. Hence, the ability to quantitate results of the pp65 assay allows the potential to stratify the risk of progression from asymptomatic infection to symptomatic disease based upon the height of the antigenemia assay. A positive assay result is the identification of anywhere from 1 to $>1000$ positively staining nuclei per $2 \times 10^{5}$ leukocytes. In general it can be concluded that the greater the value of the antigenemia assay, the higher the risk of progression to symptomatic disease. Further experience in SOT patients has demonstrated that the assay becomes positive days to weeks in advance of clinical symptoms; ${ }^{[27,28,30,32]}$ therefore, this test should be able to serve as the basis for initiation of pre-emptive treatment of asymptomatic CMV infection. 


\subsection{Polymerase Chain Reaction (PCR)}

Nucleic acid amplification by PCR on DNA or RNA extracted from infected leukocytes allows the rapid diagnosis (same day) of CMV infection. ${ }^{[33,34]}$ Also, PCR techniques can detect CMV DNA in whole blood ${ }^{[35]}$ and cell-free body fluids such as serum and plasma. ${ }^{[36,37]}$ Unfortunately, although simple qualitative PCR techniques are very sensitive and become positive several weeks prior to the onset of symptoms, the positive predictive value of these assays for the development of disease is only around 50\% ${ }^{[38,39]}$ In an effort to improve specificity, quantitative PCR assays for the measurement of the CMV viral load in the peripheral blood leukocytes have been developed to be used as a guide for pre-emptive therapy. ${ }^{[40]}$ Additional amplification strategies to PCR have been developed and include the hybrid capture assay, ${ }^{[41]}$ the branched DNA assay, ${ }^{[42]}$ and nucleic acid sequencebased amplification. ${ }^{[43,44]}$ Each of these strategies serves as an alternative quantitative test to detect CMV DNA or RNA in whole blood or leukocytes.

\section{Prevention of CMV Disease}

Over the last decade, three major strategies have been developed for the prevention of CMV disease after transplantation: (i) reduction of risk of viral acquisition or reactivation by management of two risk factors (donor/recipient serological status, and blood transfusion); (ii) prophylaxis of all 'at-risk' patients (using either an antiviral agent or an intravenous immunoglobulin product) for a defined period of time, initiated near the time of transplantation; and (iii) pre-emptive treatment with ganciclovir of selected patients guided by either laboratory markers indicative of subclinical infection or the presence of specific risk factors (e.g. exposure to augmented immunosuppression). While a growing amount of literature describes these approaches in adult transplant recipients, published experience in children has been much more limited. A consensus does not currently exist concerning whether prophylactic or pre-emptive therapy is the optimal strategy for preventing CMV disease. Further work is needed to identify whether specific preventive strategies will work equally well in recipients of different types of transplantation. This is particularly true for recipients of intestinal, lung and BMT in whom the consequences of CMV disease appear to be greater than other types of transplantation.

\subsection{Reduction of Risk of Viral Acquisition}

Many children undergoing transplantation are 'immunologically naive' to CMV and, therefore, are at high risk of acquiring a primary infection. An obvious strategy for the prevention of
CMV disease in these children would be to only use organs from seronegative donors. In general, this approach is not practical because of the limited pool of seronegative donors; however, this policy has been recommended for $\mathrm{CMV}$-seronegative candidates awaiting isolated intestine allografts in whom the transplant procedure may be deemed to be 'elective', and in whom the outcome of primary CMV infection continues to be associated with unacceptable morbidity and mortality. ${ }^{[45]}$ This approach has also been adopted by some pediatric lung transplant centers. One way that centers might maximize their chances of obtaining seronegative donors without necessarily requiring the use of a CMVseronegative donor for CMV-seronegative candidates would be to consider a strategy of early listing of patients at high risk, allowing them the freedom to be 'selective' when donor offers are made to their candidates.

A second and uniformly available approach to reduce the risk of acquiring $\mathrm{CMV}$ is through the use of CMV-seronegative blood products during the transplant operation and throughout the posttransplant course. The use of CMV-seronegative blood products has been shown to be effective in preventing CMV infection in CMV-seronegative recipients of BMT and SOT. ${ }^{[46]}$ When CMVnegative blood products are not available, CMV-safe (e.g. filtered or leukocyte-poor) blood products should be used. While neither of these approaches is perfect, they are associated with a failure rate of only 1 to $4 \%$.[47]

\subsection{Prophylaxis of All At-Risk Patients}

\subsubsection{Active Immunoprophylaxis}

The ideal intervention for preventing CMV infection after transplantation would be active immunization of seronegative recipients with a well tolerated, immunogenic vaccine; however, efforts to date have not yet identified the appropriate candidate vaccine. Early efforts at vaccine development utilized the live, attenuated CMV Towne strain. This candidate vaccine was used in three randomized, placebo-controlled studies in adult renal transplant recipients. ${ }^{[48-50]}$ Although the vaccine appeared to be well tolerated, it did not alter the incidence of CMV infection in seronegative recipients of kidneys from seropositive donors; however, use of the vaccine did appear to modify the severity of CMV disease in these patients. Recent efforts have focused on subunit glycoprotein $\mathrm{B}$ and $\mathrm{H}$ candidate vaccines. ${ }^{[51,52]}$ The best studied is the glycoprotein $\mathrm{B}$, which is responsible for at least one-half of neutralizing antibodies in the serum of naturally infected individuals. ${ }^{[52]}$ A glycoprotein B candidate vaccine has been engineered which is immunogenic in healthy adults and toddlers. ${ }^{[53]}$ Although a trial in dialysis patients yielded apparently promising results, it was discontinued prior to completion several 
years ago. A canarypox vector-expressing CMV pp65 is another potential candidate vaccine which has been shown to induce longlasting cytotoxic $\mathrm{T}$ cells in $\mathrm{CMV}$-seronegative adult volunteers. ${ }^{[54]}$ While each of these candidates is promising, none of them is currently close to licensure nor have they been evaluated in children with end-stage organ disease.

\subsubsection{Passive Immunoprophylaxis}

CMV hyperimmune globulin (CMVIG) is standardized to provide an approximately 5-fold enrichment in anti-CMV titer as compared with unselected immune globulin (IVIG). ${ }^{[55]}$ While some data exist supporting a prophylactic effect of standard IVIG in the prevention of CMV in SOT recipients, ${ }^{[56-58]}$ the use of CMVIG is thought to provide superior levels of protection compared with standard preparations in this patient population. To date, however, no study has directly compared the effect of CMVIG with that of IVIG in the transplant population. Approved prophylactic regimens using CMVIG call for an initial infusion at the time of transplant, with subsequent doses at 2, 4, 6, 8, 12 and 16 weeks post-transplant. CMVIG has been shown to prevent CMV disease in both high and low risk liver transplant recipients, ${ }^{[59-61]}$ and has been shown to reduce the rate of CMV disease by $\geq 50 \%$ in kidney transplant recipients at risk of primary CMV disease. ${ }^{[62]}$ A metaanalysis appeared to confirm the efficacy of CMVIG after SOT, ${ }^{[63]}$ and its use has also been associated with increased survival after liver transplantation, ${ }^{[64]}$ as well as the reduction of non-CMV opportunistic infection following SOT in general. ${ }^{[59]}$ In contrast to the demonstrated efficacy in SOT recipients, the use of CMVIG in BMT is controversial. Some authors have reported efficacy whereas others have not. ${ }^{[65,66]}$ Accordingly, recommendations for its routine use after BMT have no clear basis at the present time. Finally, the main disadvantage of CMVIG is the high cost.

\subsubsection{Chemoprophylaxis}

Chemoprophylactic strategies are based on the use of an antiviral agent initiated at or near the time of transplant in order to prevent CMV infection or suppress its reactivation. Seronegative recipients who receive organs from a seronegative donor should not receive chemoprophylaxis. In general, studies of chemoprophylaxis against the development of CMV disease have evaluated high dose oral aciclovir, valaciclovir, intravenous ganciclovir and oral ganciclovir. Table II summarizes general aspects of those drugs, as well as foscarnet and cidofovir. In general, well designed studies providing direct comparisons of one or more of these agents have not been carried out and data regarding the use of prophylactic antiviral agents in SOT recipients are somewhat confusing. Couchoud et al. ${ }^{[67]}$ performed a meta-analysis of randomized trials evaluating antiviral agents in the prevention of CMV infection and disease in adults and pediatric recipients of SOT. The studies compared prophylactic treatment with aciclovir and/or ganciclovir with control groups receiving either no treatment or placebo. The meta-analysis suggested that prophylaxis with ganciclovir resulted in a decreased rate of CMV infection and disease compared with placebo or no treatment. In contrast, the use of prophylactic aciclovir was associated with a decrease in CMV disease but not CMV infection. Similarly, a recent review by Paya ${ }^{[68]}$ suggested that while use of high dosage oral aciclovir was more effective than either no treatment or placebo, the results achieved using oral aciclovir were suboptimal, particularly when compared with those achievable with ganciclovir.

While ganciclovir appears to be more effective than aciclovir, its tolerability profile is less optimal and its use may require central line placement to provide long-term therapy. Bone marrow and renal toxicity are the most frequent adverse effects. This is a particular concern in recipients of allogeneic BMT, in whom ganciclovir-related neutropenia has been associated with bacte-

Table II. Characteristics of antiviral drugs

\begin{tabular}{|c|c|c|c|c|}
\hline Antiviral drug & $\begin{array}{l}\text { In vitro } \\
\text { activity }\end{array}$ & Advantages & Disadvantages & Principal adverse effects \\
\hline \multirow[t]{2}{*}{ Aciclovir } & + & Oral & Low bioavailability & Neuro- and nephrotoxicity \\
\hline & & Inexpensive and well tolerated & & Gastrointestinal symptoms \\
\hline Valaciclovir & ++ & Oral & Greater bioavailability than aciclovir & Neurotoxicity (hallucinations) \\
\hline \multirow[t]{2}{*}{ Intravenous ganciclovir } & +++ & Proven efficacy & Long-term use & Bone marrow suppression \\
\hline & & & Requires central venous access & Nephrotoxicity \\
\hline \multirow[t]{3}{*}{ Oral ganciclovir } & ++ & Oral & Poor availability & Same as intravenous ganciclovir \\
\hline & & & Requires high doses & \\
\hline & & & Pharmacokinetics in children not well defined & \\
\hline Foscarnet & +++ & No bone marrow suppression & Requires probenecid and prehydration & Nephrotoxicity \\
\hline \multirow[t]{2}{*}{ Cidofovir } & ++++ & Weekly administration & Requires probenecid and prehydration & Nephrotoxicity \\
\hline & & & No clinical experience in children & Ophthalmological adverse effects \\
\hline
\end{tabular}


rial and fungal infection and late CMV disease secondary to recovery delay of CMV-specific cytotoxic T-cell function. ${ }^{[69-71]}$ Finally, although antiviral resistance among strains of CMV in transplant recipients has been described, ${ }^{[72-77]}$ increasing use of these agents may result in diminished benefits of these prophylactic strategies. Hence, continued vigilance for changes in the prevalence of resistance to CMV will be necessary in order to assure the ongoing benefits of any chemoprophylactic approach. A brief summary of results achieved with individual agents follows below.

Aciclovir

The use of high dosage, oral aciclovir was the first widely accepted chemoprophylactic strategy for the prevention of CMV. Although several studies did demonstrate the effectiveness of this agent, ${ }^{[67,78,79]}$ subsequent studies were not consistently able to reproduce these findings, and enthusiasm for its use has waned. ${ }^{[80,81]}$ One major reason for the lack of sustained enthusiasm has been the availability of intravenous, and subsequently oral, ganciclovir. Finally, aciclovir prophylaxis has not been shown to be effective in CMV-seropositive patients receiving muromonab CD3 or antilymphocyte preparations, or high risk patients after SOT. ${ }^{[81,82]}$ Although limited data evaluating the use of intravenous aciclovir suggest a potential role in the prevention of CMV in the allogeneic BMT population, ${ }^{[83]}$ it is not effective in autologous BMT. ${ }^{[84]}$ This approach has not been widely adopted and is not recommended at this time.

\section{Valaciclovir}

This ester valine prodrug of aciclovir has greater bioavailability and hence, results in higher serum levels than oral aciclovir. ${ }^{[85]}$ Consequently, valaciclovir should offer more effective oral prophylaxis compared with oral aciclovir. Use of valaciclovir for 90 days significantly reduced the risk and delayed the onset of CMV disease, herpes simplex virus infection, and acute graft rejection compared with a placebo control in renal transplant recipients. ${ }^{[86]}$ In contrast, other investigators found that the use of valaciclovir was not cost effective in CMV D+/R- recipients of renal transplant compared with placebo. ${ }^{[87]}$ The use of valaciclovir for patients who had undergone allogeneic BMT was equivalent to results achieved with high dosage aciclovir prophylaxis. ${ }^{[88]}$

\section{Intravenous Ganciclovir}

Two meta-analyses suggest that ganciclovir is an efficacious prophylactic agent against CMV disease in SOT. ${ }^{[67,89]}$ Furthermore, the use of intravenous ganciclovir protected against the development of CMV disease in adult liver transplant recipients receiving muromonab $\mathrm{CD} 3 .{ }^{[90]}$ What remains unclear is the optimal duration and route of ganciclovir prophylaxis in this population. Published experience suggests that different regimens will likely be necessary for different types of patients with varying levels of risk of developing CMV disease (e.g. seropositive liver transplant recipients versus CMV D+/R-intestinal transplant recipients). Intravenous ganciclovir administered for 100 days has been shown to be effective in preventing CMV disease after both liver transplantation and allogeneic BMT. ${ }^{[70,71]}$ Nevertheless, its use does not impact on overall survival after BMT, maybe because of myelosuppression and reconstitution delays of immune function. In contrast, Patel et al. ${ }^{[60]}$ concluded that shorter courses ( 2 to 4 weeks) of intravenous ganciclovir should be beneficial in non-high risk heart and liver transplant recipients (and probably in high risk renal transplant recipients); however, longer courses of intravenous therapy may be necessary to prevent CMV disease in high risk populations, including at-risk lung (e.g. all but D-/R-) and $\mathrm{D}+/ \mathrm{R}-$ heart and liver transplant recipients. ${ }^{[91-93]}$ In some scenarios, data are contradictory as to what the appropriate duration of intravenous therapy should be. For example, while some studies have suggested that short course therapy is not adequate for high risk liver transplant recipients, ${ }^{[60]}$ use of 14 days of intravenous ganciclovir prophylaxis resulted in good outcomes in pediatric liver transplant recipients. ${ }^{[94]}$ Head-to-head studies comparing short versus long courses of intravenous ganciclovir prophylaxis will be necessary to resolve these contradictions. In addition, consensus must be achieved on what the appropriate endpoints (e.g. prevention of all CMV infection versus preventing CMV disease) should be prior to the initiation of such studies.

\section{Oral Ganciclovir}

Oral ganciclovir has a relatively low mean bioavailability in humans (6 to 9\%), which is more pronounced in children. A multicenter study in adult recipients of liver allografts found that the incidence of CMV disease at 6 months was $18.9 \%$ with placebo versus $4.8 \%$ with oral ganciclovir. ${ }^{[95]}$ Disease was also prevented in this study in the high risk $(\mathrm{D}+/ \mathrm{R}-)$ population. Oral ganciclovir has also been found to be more effective than oral aciclovir in D+/R- kidney transplant recipients. ${ }^{[96]}$ Unfortunately, the pharmacokinetics, tolerability, tolerance and antiviral effects of oral ganciclovir in children have not been well defined. Three studies of the pharmacokinetics of oral ganciclovir in children have been reported; two of them after transplantation (table III). ${ }^{[97-99]}$ Although minimal toxicity was reported, intolerance of the large volume of suspension or numerous capsules was the primary reason for discontinuation of ganciclovir. The results from these studies suggest that a dosage of oral ganciclovir $\geq 30$ $\mathrm{mg} / \mathrm{kg}$ every 8 hours should provide effective prophylaxis of CMV disease in children. One study also presented a method to calculate the ganciclovir dose for children with an impaired renal allograft. 
Table III. Pharmacokinetic studies of oral ganciclovir in children

\begin{tabular}{|c|c|c|c|}
\hline Recommended dosage & No. of patients and study population & $\begin{array}{l}\text { CMV effective } \\
\text { prophylaxis }\end{array}$ & Reference \\
\hline $30 \mathrm{mg} / \mathrm{kg} / 8 \mathrm{~h}$ & 9 high risk $\mathrm{CMV}$ kidney and liver transplant recipients & Yes & Frenkel et al. $^{[97]}$ \\
\hline $20-40 \mathrm{mg} / \mathrm{kg} / 8 \mathrm{~h}$ & 36 children infected with HIV & Yes & Pescovitz et al. ${ }^{[98]}$ \\
\hline $100 \mathrm{mg} / \mathrm{kg} /$ day in 3 doses & $14 \mathrm{CMV}$ D+/R- kidney transplant recipients & Yes & Filler et al. ${ }^{[99]}$ \\
\hline
\end{tabular}

Valganciclovir

Valganciclovir is a valine ester of ganciclovir that has recently been approved for use in patients with AIDS. As in the case of valaciclovir, the addition of the valine moiety results in a dramatic improvement in the bioavailability of the parent drug. Preliminary experience in liver transplant recipients has been promising. ${ }^{[100]}$

\section{Foscarnet}

This pyrophosphate analog does not cause myelosuppression but is associated with significant nephrotoxicity. Use of foscarnet should be reserved for ganciclovir-resistant CMV disease, ${ }^{[101]}$ although clinicians must be aware that cross-resistance between foscarnet and ganciclovir (and rarely even cidofovir) has been reported. ${ }^{[73,75]}$ Because of its lack of bone marrow suppression, foscarnet has been evaluated and found effective for CMV prophylaxis following allogeneic BMT in adult patients who were unable to receive ganciclovir. ${ }^{[102]}$ It has also been used with success for pre-emptive therapy in allogeneic BMT and hemopoietic stem cell recipients, alone or in combination with ganciclovir, respectively. ${ }^{[103,104]}$

\section{Cidofovir}

Cidofovir is a nucleotide analog, 10 -fold more potent in vitro than ganciclovir. ${ }^{[105]}$ The drug has a long intracellular half-life ( $>48$ hours) and may inhibit CMV for 5 to 10 days, which allows for weekly administration. ${ }^{[106]}$ It has efficacy in CMV retinitis in patients with AIDS. ${ }^{[107]}$ Occasionally cidofovir has been used in the pediatric population, and it may hold promise for improving the treatment of pediatric viral infection. ${ }^{[108]}$ Like foscarnet, the high frequency of nephrotoxicity associated with the use of cidofovir limits its use to clinical scenarios suggestive of ganciclovir resistance; however, cidofovir resistance has been described after allogeneic BMT in children. ${ }^{[73]}$ Ophthalmological adverse effects have also been reported with cidofovir. ${ }^{[109]}$

\subsection{Pre-emptive Therapy}

The aim of pre-emptive therapy is to identify and treat patients with subclinical CMV infection before they progress to symptomatic disease. ${ }^{[110]}$ The strategy depends upon the use of laboratory markers or clinical characteristics to identify patients at high risk of developing disease prior to the onset of symptoms. The first laboratory marker used for pre-emptive therapy was with viral cultures using the shell vial assay. ${ }^{[70,111]}$ Unfortunately, positive cultures in CMV-seropositive transplant recipients have not always accurately predicted progression from asymptomatic infection to CMV disease. ${ }^{[112,113]}$ More recently, pre-emptive strategies have relied on quantitation in the peripheral blood of CMVpp65 antigen and/or the PCR assay. ${ }^{[28,40]}$

\subsubsection{CMV-pp65 Antigenemia Assay}

Despite the fact that there is extensive literature about the CMV-pp65 assay, strict guidelines for its use as a guide to preemptive therapy have not been agreed upon. Suggested thresholds for initiation of pre-emptive treatment have not been standardized and have varied among centers and different types of transplant recipients. For SOT, most clinicians believe that for CMVseronegative pretransplant recipients, any positive result (pp65 antigenemia $\geq 1$ ) indicates primary infection and is an indication for pre-emptive therapy. In contrast, there is no 'gold standard' for patients who were CMV-seropositive prior to SOT. Studies in heart transplant recipients suggested that patients with an assay of $\geq 100 / 2 \times 10^{5}$ leukocytes might benefit from pre-emptive therapy. ${ }^{[32]}$ In contrast, Kusne et al. ${ }^{[28]}$ have suggested that pre-emptive therapy should be initiated for seropositive SOT recipients when the antigenemia assay is $>10 / 2 \times 10^{5}$ leukocytes, because that threshold was associated with a 7-fold increase in the risk of developing CMV disease in a retrospective analysis of seropositive adult liver transplant recipients. For BMT recipients, in addition to the CMV serostatus of the recipient and the donor, whether the patient underwent an autologous or allogeneic BMT also has to be taken into consideration. One recent study reported that only 10 to $15 \%$ of CMV-seropositive recipients of autologous BMT with antigenemia will progress to CMV disease compared with 50 to $60 \%$ of seropositive recipients of allogeneic BMT with antigenemia. ${ }^{[113]}$ It was suggested that recipients of autologous transplants with $>5$ positive cells/1.5 x $10^{5}$ leukocytes should receive preemptive therapy.

Several disadvantages of the pp65 antigenemia assay are noted in table IV. We recommend its use for patients at high risk of developing CMV disease after small bowel and lung transplan- 
Table IV. Disadvantages of the pp65 antigenemia assay

\begin{tabular}{l} 
Staining with immunoperoxidase may lead to different results than \\
indirect immunofluorescence \\
Results of tests performed on shipped specimens may not be accurate \\
Limited utility in patients with neutropenia (insufficient neutrophils to count) \\
\hline
\end{tabular}

tation, as well as for recipients of BMT. We also recommend its use in monitoring the effectiveness of the CMV disease treatment.

\subsubsection{PCR}

As is the case with the pp65 antigenemia assay, consensus on appropriate CMV PCR thresholds for initiation of pre-emptive therapy has not yet been achieved. One set of investigators has suggested using 100 genome copies $/ 10^{5}$ leukocytes as the threshold to initiate pre-emptive therapy in allogeneic BMT patients, as well as in SOT recipients experiencing primary CMV infection; ${ }^{[40,114]}$ however, they recommend a higher cutoff of 1000 genome copies $/ 10^{5}$ leukocytes when monitoring CMV-seropositive SOT recipients. ${ }^{[115]}$ Prospective studies confirming the appropriateness of these proposed cutoffs have not been carried out. Additional limitations to the use of quantitative CMV PCR as a guide to initiation of pre-emptive therapy include the fact that standardization of tests between transplant centers has not been accomplished; a variety of different primer tests and quantitative strategies have been used. However, one potentially important advantage of PCR compared with the antigenemia assay is the fact the specimens can be stored safely and shipped without significantly affecting the results of the assay. ${ }^{[116,117]}$

Theoretical advantages and disadvantages of pre-emptive therapy compared with chemoprophylaxis are shown in table V. Routine prophylactic strategies result in up to $65 \%$ of patients receiving antiviral therapy unnecessarily. It is of particular interest after BMT, where the use of pre-emptive therapy is associated with a reduction in ganciclovir use, less neutropenia, earlier recovery of CMV-specific immunity and less invasive fungal infection. ${ }^{[15,118]}$ In contrast, the use of pre-emptive therapy has been associated with an increase of late CMV disease and CMV pneumonia after discontinuation of ganciclovir when antigenemia was no longer detected. ${ }^{[15,118]}$ Screening for CMV infection with antigenemia or PCR beyond day 100 seems reasonable.

\section{Treatment of CMV Infection and Disease}

Ganciclovir is the drug of choice for the treatment of CMV disease after SOT and BMT in children. Its long record of success and relatively infrequently encountered adverse effects support its use over either foscarnet or cidofovir. Bone marrow suppression may limit its use in BMT recipients. Unresolved issues regarding the treatment of CMV disease include the duration of therapy, the potential utility of combination therapy using CMVIVIG, and the value of following the pp65 antigenemia assay or quantitative CMV PCR as a guide to the duration of therapy. Because of the so-called indirect effects of CMV (e.g. chronic rejection), some investigators have felt that even asymptomatic CMV infection should be avoided. However, data do not exist to support the treatment of asymptomatic CMV infection as a means of preventing chronic rejection or late graft loss after transplantation. An overview of proposed guidelines for the treatment of CMV infection and disease is provided in table VI. Finally, while mycophenolate mofetil enhances the severity and morbidity of CMV disease after kidney transplantation, ${ }^{[119]}$ a recent study has suggested that the drug provides a protective effect against CMVmediated injury and long-term graft loss. ${ }^{[120]}$ In fact, Neyts et al. ${ }^{[121]}$ observed that mycophenolate mofetil could strongly potentiate the anti-CMV activity of aciclovir and ganciclovir, both in vitro and in vivo, in a murine model.

Table V. Theoretical advantages and disadvantages of pre-emptive versus prophylactic therapy

\begin{tabular}{ll}
\hline Pre-emptive therapy & Prophylactic therapy \\
\hline Advantages & Decrease incidence of opportunistic infections \\
Avoidance of universal prophylaxis & Prophylaxis for other herpes virus \\
Cost reduction & Proven efficacy \\
Reduction of adverse effects attributable to the use of antiviral agents & \\
Decreased likelihood of emergence of drug-resistant strains of cytomegalovirus & Universal prophylaxis \\
Disadvantages & Development of resistance \\
No well-proven advantages & \\
Intensive surveillance & \\
Logistical difficulties & \\
Patient compliance & \\
Ambiguity concerning its effect on patient care cost & \\
Standardization of testing procedures has not been accomplished &
\end{tabular}


Table VI. Proposed guidelines for the treatment of CMV infection and disease

Treatment of CMV infection and disease after solid organ transplantation

Drug of choice: intravenous ganciclovir ( $5 \mathrm{mg} / \mathrm{kg} / 12 \mathrm{~h}$ ) for 14 days (with dosage adjustment according to renal function)

If resistant to ganciclovir: intravenous ganciclovir $(5 \mathrm{mg} / \mathrm{kg} / 12 \mathrm{~h})+$ foscarnet $(90 \mathrm{mg} / \mathrm{kg} / 12 \mathrm{~h})+$ CMVIG $100 \mathrm{mg} / \mathrm{kg}$ weekly (with dosage adjustment

according to renal function)

Follow up with antigenemia or PCR

If resistant to ganciclovir and foscarnet: intravenous ganciclovir + cidofovir ( $5 \mathrm{mg} / \mathrm{kg}$ once a week for two doses, and then once every other week) + CMVIG

Follow up with antigenemia or PCR

\section{Treatment of CMV disease after bone marrow transplantation}

Drug of choice: intravenous ganciclovir ( $5 \mathrm{mg} / \mathrm{kg} / 12 \mathrm{~h}$ ) for $14-21$ days, followed by $5 \mathrm{mg} / \mathrm{kg} /$ day for at least 3-4 weeks

Marrow failure: foscarnet ( $90 \mathrm{mg} / \mathrm{kg} / 12 \mathrm{~h}$ ) for 14 days followed by $90 \mathrm{mg} / \mathrm{kg} /$ day for 2 weeks, plus G-CSF

CMV pneumonia: intravenous ganciclovir $(5 \mathrm{mg} / \mathrm{kg} / 12 \mathrm{~h}$ ) for $14-21$ days, followed for at least 3-4 weeks by $5 \mathrm{mg} / \mathrm{kg} / \mathrm{day} \mathrm{plus} \mathrm{CMVIG}$

Follow up with antigenemia or PCR

CMV = cytomegalovirus; CMVIG = cytomegalovirus hyperimmune globulin; G-CSF = granulocyte colony-stimulating factor; PCR = polymerase chain reaction.

\section{Conclusions}

Current efforts have gone beyond the treatment of CMV disease to its prevention. The major focus of these efforts have centered on prophylaxis (using either immunoprophylactic or chemoprophylactic strategies) or on pre-emptive therapy. Comparative studies are needed to investigate which strategy is the most cost effective. While some authors advocate prophylactic therapy as the preferred approach to preventing CMV disease, ${ }^{[122]}$ data directly comparing the outcomes of the two different approaches in various transplant populations are needed. The comparative efficacy of pre-emptive treatment strategies, based on either CMVpp65 antigenemia assay or quantitative CMV PCR, has not been evaluated in well designed clinical trials in either adults or children. Trials carried out in the pediatric population would be of particular interest because there is a greater proportion of children undergoing primary CMV infection (and therefore at risk of greater morbidity) than adults. Prospective trials comparing preemptive therapy using either intravenous or oral ganciclovir, and now oral valganciclovir, are necessary to determine the relative cost effectiveness and efficacy of these alternative strategies with these related agents. The use of a combined approach may be necessary for very high risk patients, e.g. D+/R-intestinal transplant recipients. Pending the availability of these proposed studies, individual centers will need to evaluate the incidence and impact of CMV disease in their transplant population to determine whether or not their current prophylactic strategies are appropriate.

\section{References}

1. Rifkind D, Starzl TE, Machioro L, et al. Transplantation pneumonia. JAMA 1964; 189 (11): 114-8

2. Stratta RJ, Shaeffer MS, Markin RS, et al. Cytomegalovirus infection and disease after liver transplantation: an overview. Dig Dis Sci 1992 May; 37 (5): 673-88
3. Singh N, Dummer JS, Kusne S, et al. Infections with cytomegalovirus and other herpes viruses in 121 liver transplant recipients: transmission by donated organ and the effect of OKT3 antibodies. J Infect Dis 1988 Jul; 158 (1): 124-31

4. Iragorri S, Pillay D, Scrine M, et al. Prospective cytomegalovirus surveillance in pediatric renal transplant patients. Pediatr Nephrol 1993 Feb; 7 (1): 55-60

5. Schowengerdt KO, Naftel DC, Seib PM, et al. Infection after pediatric heart transplantation: results of a multi-institutional study. The Pediatric Heart Transplant Study Group. J Heart Lung Transplant 1997 Dec; 16 (12): 1207-16

6. Pollard RB. Cytomegalovirus infection in renal, heart, heart-lung and liver transplantation. Pediatr Infect Dis J 1988 May; 7 Suppl. 5: 97-102

7. Bueno J, Green M, Kocoshis S, et al. Cytomegalovirus infection after intestinal transplantation in children. Clin Infect Dis 1997 Nov; 25 (5): 1078-83

8. Meyers JD, Fluornoy N, Thomas E, et al. Risk factors for cytomegalovirus infection after human bone marrow transplantation. Ann Intern Med 1993 Mar; 153 (3): 478-88

9. Falagas ME, Snydman DR. Recurrent cytomegalovirus disease in solid organ transplant recipients. Transplant Proc 1995 Oct; 27 (5 Suppl. 1): 34-7

10. Hebart H, Kanz L, Janh G, et al. Management of cytomegalovirus infection after solid-organ or stem-cell transplantation: current guidelines and future prospects. Drugs 1998 Jan; 56 (1): 59-72

11. Rubin RH. Infection in the organ transplant recipient. In: Rubin RH, Young LS, editors. Clinical approach to infection in the compromised host. 3rd ed. New York (NY): Plenum Publishing, 1994: 629-705

12. Sollinger HW. Mycophenolate mofetil for the prevention of acute rejection in primary cadaveric renal allograft recipients: US renal transplant mycophenolate mofetil study group. Transplantation 1995 Aug; 60 (3): 225-32

13. Heeman U, Azuma H, Hamar P, et al. Mycophenolate mofetil inhibits lymphocyte binding and the upregulation of adhesion molecules in acute rejection allografts. Transplant Immunol 1996 Mar; 4 (1): 64-7

14. Fishman JA, Rubin RH. Infection in organ transplant recipients. N Engl J Med 1998 Jun; 338 (24): 1741-51

15. Boeckh M, Gooley TA, Myerson D, et al. Cytomegalovirus pp65 antigenemiaguided early treatment with ganciclovir versus ganciclovir at engraftment after allogeneic transplantation. Blood 1996 Nov; 88 (10): 4063-71

16. Paya CV, Wiener RH, Hermans PE, et al. Risk factors for cytomegalovirus and severe bacterial infections following liver transplantation: a prospective multivariate time-dependent analysis. J Hepatol 1993 Jun; 18 (2): 185-95

17. Rubin RH. Impact of cytomegalovirus infections on organ transplant recipients. Rev Infect Dis 1990 Sep-Oct; 12 Suppl. 7: S754-66

18. Rubin RH. Cytomegalovirus disease and allograft loss after organ transplantation. Clin Infect Dis 1998 Apr; 26 (4): 871-3 
19. Pouteil-Noble C, Ecochard R, Landrivon R, et al. Cytomegalovirus infection, an etiological factor for rejection: a prospective study in 242 renal transplant patients. Transplantation 1993 Apr; 55 (4): 851-7

20. Bando K, Paradis IL, Similo S, et al. Obliterative bronchiolitis after lung and heart-lung transplantation: an analysis of risk factors and management. J Thorac Cardiovasc Surg 1995 Jul; 110 (1): 4-13

21. Grattan MT, Moreno-Cabral CE, Starnes VA, et al. Cytomegalovirus infection is associated with cardiac allograft rejection and atheroesclerosis. JAMA 1989 Jun; 261 (24): 3561-6

22. Arnold JC, Portmann BC, O'Grady JG, et al. Cytomegalovirus persist in the liver graft in the vanishing bile duct syndrome. Hepatology 1992 Aug; 16 (2): 494-6

23. Soderberg C, Larsson S, Rozell BL, et al. Cytomegalovirus-induced CD13specific autoimmunity: a possible cause of chronic graft-vs-host disease. Transplantation 1996 Feb; 61 (4): 600-9

24. Lonnqvist B, Ringden O, Wahren B, et al. Cytomegalovirus infection associated with and preceding chronic graft-versus-host disease. Transplantation 1984 Nov; 38 (5): 465-8

25. Barkholt L, Ehrnst A, Veress B. Clinical use of immunohistopathologic methods for the diagnosis of cytomegalovirus hepatitis in human liver allograft biopsy specimens. Scand J Gastroenterol 1994 Jun; 29 (6): 553-60

26. Paya C, Holley K, Wiesner H, et al. Early diagnosis of cytomegalovirus hepatitis in liver transplant recipients: role of immunostaining, DNA hybridization and culture of hepatic tissue. Hepatology 1990 Jul; 12 (1): 119-26

27. The TH, van der Ploeg M, van den Berg AP, et al. Direct detection of cytomegalovirus in peripheral blood leukocytes: a review of the antigenemia assay and polymerase chain reaction. Transplantation 1992 Aug; 54 (2): 193-8

28. Kusne S, Grossi P, Irish W, et al. Cytomegalovirus pp65 antigenemia monitoring as a guide for pre-emptive therapy: a cost effective strategy for prevention of cytomegalovirus disease in adult liver transplant recipients. Transplantation 1999 Oct; 68 (8): 1125-31

29. van der Bij W, Torensma R, Van Son WJ, et al. Rapid immunodiagnosis of active cytomegalovirus infection by monoclonal antibodies staining of blood leukocytes. J Med Virol 1988 Jun; 25 (2): 179-88

30. Storch GA, Buller RS, Bailey T, et al. Comparison of PCR and pp65 antigenemia assay with quantitative shell vial culture for detection of cytomegalovirus in blood leukocytes from solid organ transplant recipients. J Clin Microbiol 1994 Apr; 32 (4): 997-1003

31. Erice A, Holm MA, Gill PC, et al. Cytomegalovirus (CMV) antigenemia assay is more sensitive than shell vial cultures for rapid detection of CMV in polymorphonuclear blood leukocytes. J Clin Microbiol 1992 Nov; 30 (11): 2822-5

32. Boeckh M, Bowden RA, Goodrish JM, et al. Cytomegalovirus antigen detection in peripheral blood leukocytes after allogeneic marrow transplantation. Blood 1992 Sep; 80 (1): 1358-64

33. Jiwa NM, Van Gemert GW, Raap AK, et al. Rapid detection of human cytomegalovirus DNA in peripheral blood leukocytes of viraemic transplant recipients by the polymerase chain reaction. Transplantation 1989 Jul; 48 (1): 72-6

34. Randhawa PS, Mañez R, Frye B, et al. Circulating immediate-early mRNA in patients with cytomegalovirus infections after solid organ transplantation. J Infect Dis 1994 Nov; 170 (5): 1264-7

35. Kidd IM, Fox JC, Pillay D, et al. Provision of prognostic information in immunocompromised patients by routine application of the polymerase chain reaction for cytomegalovirus. Transplantation 1993 Oct; 56 (4): 867-71

36. Evans PC, Soin A, Wreghitt TG, et al. Qualitative and semiquantitative polymerase chain reaction testing for cytomegalovirus DNA in serum allows prediction of CMV related disease in liver transplant recipients. J Clin Pathol 1998 Dec; 51 (12): 914-21

37. Gerna G, Furione F, Baldanti F, et al. Comparative quantitation of human cytomegalovirus DNA in blood leukocytes and plasma of transplant and AIDS patients. J Clin Microbiol 1994 Nov; 32 (11): 2709-17

38. Abecassis MM, Koffron AJ, Kaplon B, et al. The role of PCR in the diagnosis and management of CMV in solid organ recipients: what is the predictive value for the development of disease and should PCR be used to guide antiviral therapy. Transplantation 1997 Jan; 63 (2): 275-9

39. Delgado R, Lumbreras C, Alba M, et al. Low predictive value of polymerase chain reaction for diagnosis of cytomegalovirus disease in liver transplant recipients. J Clin Microbiol 1992 Jul; 30 (7): 1876-8

40. Gerna G, Furione M, Baldanti F, et al. Quantitation of human cytomegalovirus DNA in bone marrow transplant recipients. Br J Haematol 1995 Nov; 91 (3): 674-83

41. Rollag H, Sagedal S, Holter E, et al. Diagnosis of cytomegalovirus infection in kidney transplant recipients by a quantitative RNA-DNA hybrid capture assay for cytomegalovirus DNA in leukocytes. Eur J Clin Microbiol Infect Dis 1998 Feb; 17 (2): 124-7

42. Chernoff DM, Miner RC, Hoo BS, et al. Quantification of cytomegalovirus DNA in peripheral blood leukocytes by a branched-DNA signal amplification assay. J Clin Microbiol 1997 Nov; 35 (11): 2740-5

43. Compton J. Nucleic acid sequence-based amplification. Nature 1991 Mar; 350 (6313): 91-2

44. Blok MJ, Goosens VJ, Vanherle SJ, et al. Diagnostic value of monitoring human cytomegalovirus late pp67 mRNA expression in renal allograft recipients by nucleic acid sequence-based amplification. J Clin Microbiol 1998 May; 36 (5): 1341-6

45. Reyes J, Bueno J, Kocoshis S. Current status of intestinal transplantation in children. J Pediatr Surg 1998 Feb; 33 (2): 243-54

46. Sayers MH, Anderson KC, Goodnough LT, et al. Reducing the risk for transfusiontransmitted cytomegalovirus infections. Ann Intern Med 1992 Jan; 116 (1): $55-62$

47. Strauss RG. Leukocyte-reduction to prevent transfusion-transmitted cytomegalovirus infections. Pediatr Transplant 1999; 3 Suppl. 1: 19-22

48. Balfour Jr HH, Sachs GW, Welo PK, et al. Cytomegalovirus vaccine in renal transplant candidates: progress report of a randomized, placebo controlled, double-blind trial. Birth Defects Orig Artic Ser 1984; 20 (1): 289-304

49. Plotkin SA, Starr SE, Friedman HM, et al. Effect of Towne liver virus vaccine on cytomegalovirus disease after renal transplant: a controlled trial. Ann Intern Med 1991 Apr; 114 (7): 525-31

50. Plotkin SA, Higgins R, Kurtz JB. Multicenter trial of Towne strain attenuated virus vaccine in seronegative renal transplant recipients. Transplantation $1994 \mathrm{Dec}$; 58 (11): 1176-8

51. Urban M, Klein M, Britt WJ, et al. Glycoprotein $H$ of human cytomegalovirus is a major antigen for the neutralizing humoral immune response. J Gen Virol 1996 Jul; 77 (Pt 7): 1537-47

52. Britt WJ, Mach M. Human cytomegalovirus glycoproteins. Intervirology 1996; 39 (5-6): 401-12

53. Plotkin SA. Vaccination against cytomegalovirus, the changeling demon. Pediatr Infect Dis 1999 Apr; 18 (4): 313-25

54. Berencsi K, Gyulai Z, Gonczol E, et al. A canarypox vector-expressing cytomegalovirus phosphoprotein 65 induces long-lasting cytotoxic T cell responses in human CMV-seronegative patients. J Infect Dis 2001 Apr 15; 183 (8): 1171-9

55. Snydman DR, McIver I, Leszczynski J, et al. A pilot trial of a novel cytomegalovirus immune globulin in liver transplant recipients. Transplantation 1984 Nov; 38 (5): 553-7

56. Glowacki LS, Smaill FM. Use of immune globulin to prevent symptomatic cytomegalovirus disease in transplant recipients: a meta-analysis. Clin Transplant 1994 Feb; 8 (1): 10-8

57. King S, Superina R, Andrews W, et al. Randomized comparison of ganciclovir plus intravenous immune globulin (IVIG) with IVIG alone for prevention of primary CMV disease in children receiving liver transplant. Clin Infect Dis 1997 Nov; 25: 1173-9

58. Flynn JT, Kaiser BA, Long SS, et al. Intravenous immune globulin prophylaxis of cytomegalovirus infection in pediatric renal transplant recipients. Am J Nephrol 1997; 17 (2): 146-52

59. Snydman DR, Werner BG, Dougherty NN, et al. Cytomegalovirus immune globulin prophylaxis in liver transplantation: a randomized, double-blind, placebo- 
controlled trial. The Boston Center for Liver Transplantation CMVIG Study Group. Ann Intern Med 1993 Nov; 119 (10): 984-17

60. Patel R, Snydman DR, Rubin RH, et al. Cytomegalovirus prophylaxis in solid organ transplant recipients. Transplantation 1996 May; 61 (9): 1279-89

61. Snydman DR. CMV prophylaxis strategies in high risk transplantation. Transplant Proc 1994 Oct; 26 (5 Suppl. L): 120-2

62. Snydman DR, Werner BG, Heinze-Lacey B, et al. Use of cytomegalovirus immune globulin to prevent cytomegalovirus disease in renal transplant recipients. $\mathrm{N}$ Engl J Med 1987 Oct; 317 (17): 1049-54

63. Wittes JT, Kelly A, Plante KM. Meta-analysis of CMVIG for the prevention and treatment of CMV infection in transplant recipients. Transplant Proc 1996 Dec; 28 (6 Suppl. 2): 17-24

64. Falagas ME, Snydman DR, Ruthazer R, et al. Cytomegalovirus immune globulin (CMVIG) prophylaxis is associated with increased survival after orthotopic liver transplantation. The Boston Center for Liver Transplantation CMVIG Study Group. Clin Transplant 1997 Oct; 11 (5 Pt 1): 432-7

65. Bowden RA, Sayers M, Flournoy N, et al. Cytomegalovirus immune globulin and seronegative blood products to prevent cytomegalovirus infection after marrow transplantation. N Engl J Med 1986 Apr; 314 (16): 1006-10

66. Meyers JD. Prevention of cytomegalovirus infection after marrow transplantation. Rev Infect Dis 1989 Nov-Dec; 11 Suppl. 7: 1691-705

67. Couchoud C, Cucherat M, Haugh M, et al. Cytomegalovirus prophylaxis with antiviral agents in solid organ transplantation: a meta analysis. Transplantation 1998 Mar 15; 65 (5): 641-7

68. Paya C. Prevention of cytomegalovirus disease in recipients of solid-organ transplants. Clin Infect Dis 2001 Feb; 32 (4): 596-603

69. Salzberger B, Bowden RA, Hackman RC, et al. Neutropenia in allogeneic bone marrow transplant recipients receiving ganciclovir for prevention of cytomegalovirus disease: risk factors and outcome. Blood 1997 Sep; 90 (6): 2502-8

70. Goodrich JM, Mori M, Gleaves CA, et al. Early treatment with ganciclovir to prevent cytomegalovirus disease after allogeneic bone marrow transplantation. N Engl J Med 1991 Dec; 325 (23): 1601-7

71. Winston DJ, Wirin D, Shaked A, et al. Randomised comparison of ganciclovir and high-dose acyclovir for long-term cytomegalovirus prophylaxis in liver-transplant recipients. Lancet 1995 Jul; 346 (8967): 69-74

72. Smith IL, Cherrington JM, Jiles RE, et al. High-level resistance of cytomegalovirus to ganciclovir is associated with alterations in both the UL97 and DNA polymerase genes. J Infect Dis 1997 Jul; 176 (1): 69-77

73. Eckle T, Prix L, Jhan G, et al. Drug-resistant human cytomegalovirus infection in children after allogeneic stem cell transplantation may have different clinical outcomes. Blood 2000 Nov; 96 (9): 3286-9

74. Alain S, Honderlick P, Grenet D, et al. Failure of ganciclovir treatment associated with selection of a ganciclovir-resistant cytomegalovirus strain in a lung transplant recipient. Transplantation 1997 May; 63: 1533-6

75. Rosen HR, Benner KG, Flora KD, et al. Development of ganciclovir resistance during treatment of primary cytomegalovirus infection after liver transplantation. Transplantation 1997 Feb; 63: 476-8

76. Green M, Bueno J, Sigurdsson L, et al. Unique aspects of the infectious complications of intestinal transplantation. Curr Opin Organ Transplant 1999 Dec; 4 (4): $361-7$

77. Limaye AP, Corey L, Koelle DM, et al. Emergence of ganciclovir-resistant cytomegalovirus disease among recipients of solid-organ transplants. Lancet 2000 Aug 19; 356 (9230): 645-9

78. Balfour Jr HH, Chace BA, Stapleton JT, et al. A randomized placebo-controlled trial of oral acyclovir for the prevention of cytomegalovirus. N Engl J Med 1989 May; 320 (21): 1381-7

79. Saliba F, Eyraud D, Samuel D, et al. Randomized controlled trial of acyclovir for the prevention of cytomegalovirus infection and disease in liver transplant recipients. Transplant Proc 1993 Feb; 25 (1 Pt 2): 1444-5

80. Winston DJ. Prevention of cytomegalovirus disease in transplant recipients. Lancet 1995 Nov; 346 (8987): 1380-1
81. Bailey TC, Ettinger NA, Storch GA, et al. Failure of high dose oral acyclovir with or without immune globulin to prevent primary cytomegalovirus disease in recipients of solid organ transplants. Am J Med 1993 Sep; 95 (3): 273-8

82. Dunn D, Gillingham KJ, Kramer MA, et al. A prospective randomized study of acyclovir versus ganciclovir plus human immune globulin prophylaxis of cytomegalovirus infection after solid organ transplantation. Transplantation 1994 Mar; 57 (6): 876-84

83. Prentice HG, Gluckman E, Powles RL, et al. Long term survival in allogeneic bone marrow transplant recipients following acyclovir prophylaxis for CMV infection. Bone Marrow Transplant 1997 Jan; 19 (2): 129-33

84. Boeckh M, Gooley T, Reusser P, et al. Failure of high dose acyclovir for prevention of CMV disease after autologous marrow transplant. J Infect Dis 1995 Oct; 172 (4): 939-43

85. Weller S, Blum MR, Doucette M, et al. Pharmacokinetics of the acyclovir pro-drug valaciclovir after escalating single- and multiple-dose administration to normal volunteers. Clin Pharmacol Ther 1993 Dec; 54 (6): 595-605

86. Lowance D, Neumayer H, Legendre M, et al. Valacyclovir for the prevention of cytomegalovirus disease after renal transplantation. N Engl J Med 1999 May; 340 (19): 1462-70

87. Legendre CM, Norman DJ, Keating MR, et al. Valacyclovir prophylaxis of cytomegalovirus infection and disease in renal transplantation: an economic evaluation. Transplantation 2000 Nov; 70 (10): 1463-8

88. Ljungman $\mathrm{P}$, Camara R, Milpied N, et al. A randomized study of valacyclovir as prophylaxis against CMV infection and disease in BMT recipients [abstract]. Bone Marrow Transplant 1999; 23 Suppl. 1: S65

89. Gourishankar S, Wong W, Dorval M. Meta-analysis of prophylaxis of CMV disease in solid organ transplantation: is ganciclovir a superior agent to acyclovir. Transplant Proc 2001 Feb-Mar; 33 (1-2): 1870-2

90. Winston DJ, Imagawa DK, Holt CD, et al. Long-term ganciclovir prophylaxis eliminates serious cytomegalovirus disease in liver transplant recipients receiving OKT3 therapy for rejection. Transplantation 1995 Dec; 60: 1357-60

91. Merigan TC, Renlund DG, Keay S, et al. A controlled trial of ganciclovir to prevent cytomegalovirus disease after heart transplantation. N Engl J Med 1992 Apr; 326 (18): 1182-6

92. Bailey TC, Trulock EP, Ettinger NA, et al. Failure of prophylactic ganciclovir to prevent cytomegalovirus disease in recipients of lung transplantation. J Infect Dis 1992 Mar; 165 (3): 548-52

93. Martin M, Mañez R, Linden P, et al. A prospective randomized trial comparing sequential ganciclovir-high dose acyclovir to high dose acyclovir for prevention of cytomegalovirus in adult transplant recipients. Transplantation 1994 Oct; 58 (7): 252-61

94. Green M, Reyes J, Nour B, et al. Randomized trial of ganciclovir followed by high dose oral acyclovir vs ganciclovir alone in the prevention of cytomegalovirus disease in pediatric liver transplant recipients: preliminary analysis. Transplant Proc 1994 Feb; 26: 173-4

95. Gane E, Saliba F, Valdecasas GJ, et al. Randomised trial of efficacy and safety oral ganciclovir in the prevention of cytomegalovirus disease in liver-transplant recipients. The Oral Ganciclovir International Transplantation Study Group. Lancet 1997 Dec; 350 (9093): 1729-33

96. Flechner SM, Avery RK, Fisher R, et al. A randomized prospective controlled trial of oral acyclovir versus oral ganciclovir for cytomegalovirus prophylaxis in high-risk kidney transplant recipients. Transplantation 1998 Dec; 66 (12): 1682-8

97. Frenkel LM, Capparelli EV, Danker WM, et al. Oral ganciclovir in children: pharmacokinetics, safety, tolerance, and antiviral effects. The Pediatric AIDS Clinical Trials Group. J Infect Dis 2000 Dec; 182 (6): 1616-24

98. Pescovitz MD, Brook B, Jindal RM, et al. Oral ganciclovir in pediatric transplant recipients: a pharmacokinetic study. Clin Transplant 1997 Dec; 11 (6): 613-7

99. Filler G, Lampe D, von Bredow MA, et al. Prophylactic oral ganciclovir after renal transplantation: dosing and pharmacokinetics. Pediatr Nephrol 1998 Jan; 12 (1): 6-9 
100. Pescovitz MD, Rabkin J, Merion RM. Valganciclovir results in improved oral absorption of ganciclovir in liver transplant recipients. Antimicrob Agents Chemother 2000 Oct; 44 (10): 2811-5

101. Manion DJ, Vibhagool A, Chou T-C, et al. Susceptibility of human cytomegalovirus to two drug combinations in vitro. Antivir Ther 1996 Dec; 1 (4): 237-45

102. Ippoliti C, Morgan A, Warkentin D, et al. Foscarnet for prevention of cytomegalovirus infection in allogeneic marrow transplant recipients. Bone Marrow Transplant 1997 Sep; 20: 491-5

103. Ljungman $\mathrm{P}$, Oberg $\mathrm{G}$, Aschan $\mathrm{J}$, et al. Foscarnet for preemptive therapy of CMV infection detected by a leukocyte-based nested PCR in allogeneic bone marrow transplant patients. Bone Marrow Transplant 1996 Sep; 18 (3): 565-8

104. Bacigalupo A, Bregante S, Tedone E, et al. Combined foscarnet-ganciclovir treatment for cytomegalovirus infection after allogeneic hemopoietic stem cell transplantation (HSCT). Bone Marrow Transplant. 1996 Nov; 18 Suppl. 2: $110-4$

105. Bronson J, Ghazzouli I, Hitchcock M, et al. Synthesis and antiviral activity of the nucleotide analogue (S)-1-(hydroxy-2-(phosphonylmethoxy)propyl)cytosine. J Med Chem 1989 Jul; 32 (7): 1457-63

106. Polis MA, Spooner K, Baird B, et al. Anticytomegaloviral activity and safety of cidofovir in patients with human immunodeficiency virus infection and cytomegalovirus viruria. Antimicrob Agents Chemother 1995 Apr; 39 (4): 882-6

107. Lalezari JP, Stagg RJ, Kupperman BD, et al. Intravenous cidofovir for peripheral cytomegalovirus retinitis in patients with AIDS: a randomized controlled trial. Ann Intern Med 1997 Feb 15; 126: 257-63

108. Legrand F, Berrebi D, Houhou N, et al. Early diagnosis of adenovirus infection and treatment with cidofovir after bone marrow transplantation in children. Bone Marrow Transplant 2001 Mar; 27 (6): 621-6

109. Akler ME, Johnson DW, Burman WJ, et al. Anterior uveitis and hipotony after intravenous cidofovir for the treatment of cytomegalovirus retinitis. Ophtalmology 1998 Apr; 105 (4): 651-7

110. Rubin RH. Preemptive therapy in immunocompromised hosts. N Engl J Med 1991 Apr; 324 (15): 1057-9

111. Schmidt GM, Horac DA, Niland JC, et al. A randomized, controlled trial of prophylactic ganciclovir for cytomegalovirus pulmonary infection in recipients of allogeneic bone marrow transplants: the city of hope. Stanford-Syntex CMV Study Group. N Engl J Med 1991 Apr; 324 (15): 1005-11

112. Falagas ME, Snydman DR, Ruthazer R, et al. Surveillance cultures of blood, urine, and throat specimens are not valuable for predicting cytomegalovirus disease in liver transplant recipients. Boston Center for Liver Transplantation CMVIG Study Group. Clin Infect Dis 1997 May; 24 (5): 824-9
113. Boeckh M, Stevens-Ayers T, Bowden RA. Cytomegalovirus pp65 after autologous marrow and peripheral blood stem cell transplantation. J Infect Dis 1996 Nov; 174 (5): 907-12

114. Gerna G, Zavattoni M, Baldanti F, et al. Human cytomegalovirus (HCMV) leukodnaemia correlates more closely with clinical symptoms than antigenemia and viremia in heart ad heart-lung transplant recipients with primary HCMV infection. Transplantation 1998 May; 65 (10): 1378-85

115. Gerna G, Baldanti F, Middeldorp J, et al. Clinical significance of expression of human cytomegalovirus pp67 late transcript in heart, lung, and bone marrow transplant recipients, as determined by nucleic acid sequence-based amplification. J Clin Microbiol 1999 Apr; 37 (4): 902-11

116. Grundy JE, Ehrnst A, Einsele H, et al. A three-center European external quality control study of PCR for the detection of cytomegalovirus DNA in blood. J Clin Microbiol 1996 May; 34 (5): 166-70

117. Schafer P, Tenschert W, Gutensohn K, et al. Minimal effect of delayed sample processing on results of quantitative PCR for cytomegalovirus DNA in leukocytes compared to results of an antigenemia assay. J Clin Microbiol 1997 Mar; 35 (3): $741-4$

118. Einsele H, Ehninger G, Hebart H, et al. Polymerase chain reaction monitoring reduces the incidence of cytomegalovirus disease and the duration and side effects of antiviral therapy after bone marrow transplantation. Blood 1995 Oct; 86 (7): 2815-20

119. Sarmiento JM, Dockrell DH, Schwab TR, et al. Mycophenolate mofetil increases cytomegalovirus invasive disease in renal transplant patients. Clin Transplant 2000 Apr; 14 (2): 136-8

120. Giral M, Nguyen JM, Daguin P, et al. Mycophenolate mofetil does not modify the incidence of cytomegalovirus disease after kidney transplantation but prevents CMV-induced chronic graft dysfunction. J Am Soc Nephrol 2001 Aug; 12 (8): 1758-63

121. Neyts J, Andrei G, De Clerq E. The novel immunosuppressive agent mycophenolate mofetil markedly potentiates the antiherpes-virus activities of acyclovir, ganciclovir, and penciclovir in vitro and in vivo. Antimicrob Agents Chemother 1998 Feb; 42 (2): 216-22

122. Hart GD, Paya CV. Prophylaxis for CMV should now replace pre-emptive therapy in SOT. Rev Med Virol 2001 Mar-Apr; 11 (2): 73-81

Correspondence and offprints: Dr Javier Bueno, Unidad de Trasplante Pediátrico, Servicio de Cirugía Pediátrica, Complejo Hospitalario Juan Canalejo, As Xubias 86, A Coruña 15006, Spain.

E-mail: jbueno@canalejo.org 